\title{
A Rapid Weak Signal Detection Approach in Resonant Pressure Sensors
}

\author{
Yanqin Zhang ${ }^{1, a}$, Weiwei Xing ${ }^{1, b}$ and Chenxiao Zhang ${ }^{1, c}$ \\ ${ }^{1}$ School of Instrumentation Science and Opto-electronics Engineering, Beihang University, Beijing \\ 100191, China. \\ akhzyq@163.com, balex.xing@163.com, zhangcx@buaa.edu.cn
}

Keywords: resonant pressure sensor, weak signal detection, cross-correlation detection, linear fit

\begin{abstract}
In this paper, a rapid detection method for weak signal detection is proposed in resonant pressure sensors. The method is based on cross-correlation with a dedicated phase sensitive detector (PSD) and an improved integrator. The PSD is the sensitive resistor which is an ideal analog multiplier based on Ohm's law. The AC voltage applied to the resistor is modulated by square wave so that the input of improved integrator is signal with alternate inverting phase. The low frequency noise is proved to be eliminated via difference of integrator output and the high frequency noise can be suppressed efficaciously by linear fit. The prototype is made and the experiment results show that the rapid cross-correlation detection has larger signal noise improvement ratio (SNIR) even though its measurement time is $1 / 25$ of the traditional LIA.
\end{abstract}

\section{Introduction}

Micro-machined resonant sensors work on the principle that the resonant frequency can be changed by resonators' stiffness. To measure the resonant frequency, the resonator needs to be excited into vibration and the vibration needs to be detected. Weak signal detection ought to be introduced to obtain such weak vibration as the dimension of resonator is typically micro-meters. ${ }^{[1][2]}$ Lock-in amplifier (LIA) ${ }^{[3][4]}$ and cross-correlation detection method ${ }^{[5][6]}$ are effective ways to detect weak periodic signal, but the improvement of their SNIR is at the cost of measurement time. Rapid cross-correlation detection is put forward in this paper in order to obtain high SNIR in short time.

\section{Theory of Rapid Cross-Correlation Detection}

\section{Rapid Cross-Correlation Model.}

The rapid cross-correlation detection is based on the traditional cross-correlation detection whose. principle is shown in Fig.1. $x(t)$ is the signal to be measured, $y(t)$ is the reference signal whose frequency is set in advance. With the multiplication of $x(t)$ and $y(t)$, the DC part will be obtained by integrator. The output cross-correlation function $R_{\mathrm{xy}}(t)$ contains the amplitude of the useful signal.

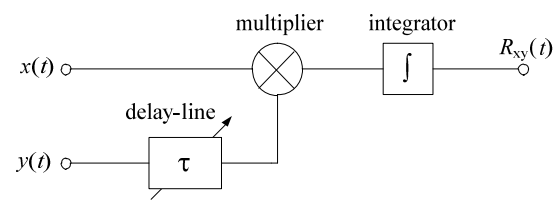

Fig.1 Schematic of cross-correlation detection

In order to calculate the value of cross-correlation function accurately, infinite integral time is necessary in theory. But in fact, limited integral time is allowed for the need of rapidity:

$$
\hat{R}_{x y}(\tau)=\frac{1}{T} \int_{0}^{T} x(t) y(t-\tau) \mathrm{d} t
$$

Fig. 2 shows the schematic of rapid cross-correlation detection. Different from the traditional cross-correlation detection, it uses a detective resistor as the multiplier; it uses an integrator which has the controllable integration time to replace the ordinary integrator, and uses linear fit as the demodulation method. And the integration capacitor's charging and discharging is controlled by the opening and closing of switch $\mathrm{K}$. The application of a detective resistor as the multiplier based on Ohm's law is named Ohm phase sensitive detection (Ohm-PSD). 


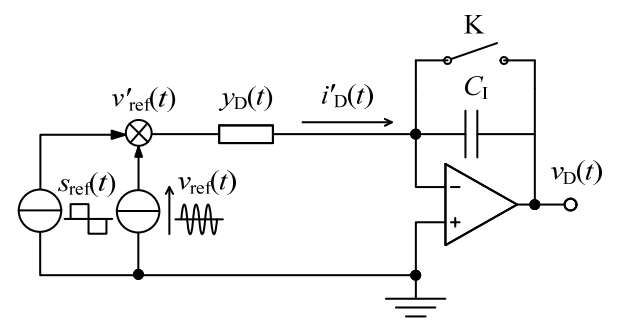

Fig.2 Principle of rapid cross-correlation detection

The conductance of the detective resistor $y_{\mathrm{D}}(t)$ is composed of an AC value and a DC bias.

$y_{\mathrm{D}}(t)=Y_{\mathrm{D}}+y_{\mathrm{d}}(t)=Y_{\mathrm{D}}+Y_{\mathrm{d}} \cos \left(\omega_{\mathrm{d}} t+\varphi_{\mathrm{d}}\right)=Y_{\mathrm{D}}-Y_{\mathrm{D}} \varepsilon_{\mathrm{d}} \cdot \cos \left(\omega_{\mathrm{d}} t+\varphi_{\mathrm{d}}\right)$

$Y_{\mathrm{D}}$ is the original conductance of detective resistor; $Y_{\mathrm{d}}$ is the peak-to-peak value of the AC part; $\omega_{\mathrm{d}}$ is the angular frequency of the $\mathrm{AC}$ part; $\varphi_{\mathrm{d}}$ is the starting phase; $\varepsilon_{\mathrm{d}}$ is the relative variable quantity of conductance.

The sine voltage signal is:

$v_{\text {ref }}(t)=V_{\text {ref }} \cos \left(\omega_{\text {ref }} t+\varphi_{\text {ref }}\right)$

In the actual detection, $\omega_{\mathrm{d}}=\omega_{\mathrm{ref}}$.

When the sine reference voltage $\nu_{\text {ref }}(t)$ is applied to the resistor and $\varphi_{\text {ref }}=\varphi_{\mathrm{d}}=0$, the current of the detective resistor is:

$$
i_{\mathrm{D}}(t)=-\frac{1}{2} V_{\text {ref }} Y_{\mathrm{D}} \varepsilon_{\mathrm{d}}-\frac{1}{2} V_{\text {ref }} Y_{\mathrm{D}} \varepsilon_{\mathrm{d}} \cos 2 \omega_{\mathrm{d}} t+V_{\text {ref }} Y_{\mathrm{D}} \cos \omega_{\text {ref }} t
$$

Equation (4) shows that the multiplication of reference signal and the measured signal can be completed expediently the moment reference voltage is applied, and this dedicated PSD is called "Ohm-PSD". Which has the advantages of large input dynamic range, strong anti-interference ability, and low component noise. Obviously, the first two items in equation (4) include the useful signal $\varepsilon_{\mathrm{d}}$, and the last item is one-time frequency interference. And the amplitude of DC part becomes largest under in-phase condition. Thus the mission of rapid cross-correlation detection is to detect its DC part, and restrain the other two parts.

For better effects of subsequent noise reduction, the reference signal $v_{\text {ref }}^{\prime}(t)$ is not pure sine signal, but modulated wave $v_{\text {ref }}^{\prime}(t)$ by square wave $s_{\text {ref }}(t)$.

$$
v_{\text {ref }}^{\prime}(t)=v_{\text {ref }}(t) \cdot s_{\text {ref }}(t)
$$

When the modulated reference voltage $v_{\text {ref }}^{\prime}(t)$ is applied to the resistor, the output current:

$$
i_{D}^{\prime}(t)= \begin{cases}+i_{\mathrm{D}}(t)=+v_{\text {ref }}(t) y_{\mathrm{D}}(t), & k T_{\mathrm{w}} \leq t \leq T_{\mathrm{C}}+k T_{\mathrm{w}} \\ -i_{\mathrm{D}}(t)=-v_{\text {ref }}(t) y_{\mathrm{D}}(t), & T_{\mathrm{C}}+k T_{\mathrm{w}} \leq t \leq T_{\mathrm{w}}+k T_{\mathrm{w}}\end{cases}
$$

Where $T_{w}=2 T_{C}, k=0,1,2 \cdots$, and $T_{w}$ is the cycle of square signal $S_{\text {ref }}(t)$.

Fig. 3 shows some important curves of rapid cross-correlation detection, where $i_{\text {DU }}^{\prime}(t)$ is part of $i_{\mathrm{D}}^{\prime}(t)$ except for one-time frequency section, and $v_{\mathrm{D}}(t)$ is an integral of $i_{\mathrm{DU}}^{\prime}(t)$.

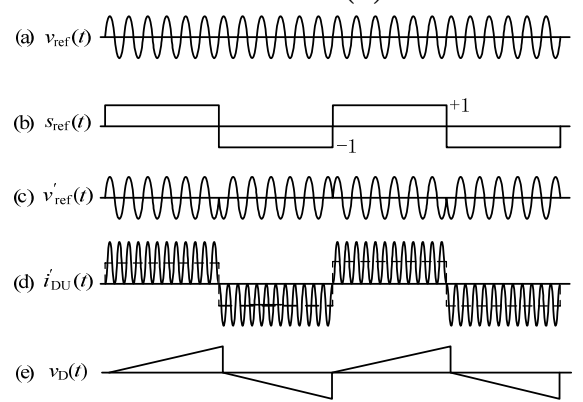

Fig. 3 Waves of rapid cross-correlation detection 
It can be seen from Fig.3 (e) that there are two integration lines with contrary slopes in one cycle of the square signal. The two integration lines are referred as $v_{D 1}(t)$ and $v_{D 2}(t)$, whose effective information is not only the instantaneous value but also the slope. When the output voltage is sampled, the linear fit is applied to obtain its slope, which is called linear fit demodulation. So the output of the rapid cross-correlation detection system is the difference between the two slopes, $v_{D 1}(t)$ and $v_{D 2}(t)$.

Noise analysis.

The electronic components (integrator, resistor, etc.) used in the rapid cross-correlation detection are not ideal components because of component noise ${ }^{[7][8]}$ such as thermal noise, shot noise, flicker noise, burst noise, etc. Fig.4 shows its noise model:

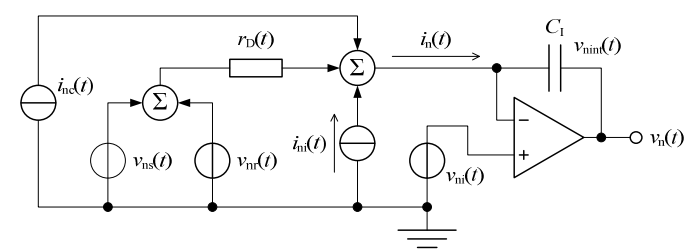

Fig.4 Noise model of rapid cross-correlation detection

When $0 \leq t \leq T_{\mathrm{C}}$, the output noise voltage is:

$v_{\mathrm{n}}\left(T_{\mathrm{I}}\right)=\frac{1}{C_{\mathrm{I}}} \int_{0}^{T_{\mathrm{I}}} i_{\mathrm{n}}(t) \mathrm{d} t$

The output noise power is:

$P_{\text {no }}=E\left\{\left[v_{\mathrm{n}}\left(T_{\mathrm{I}}\right)\right]^{2}\right\}=\frac{4}{C_{\mathrm{I}}^{2}} \int_{0}^{T_{1}} \int_{0}^{T_{1}} R_{n}(t-\tau) d t d \tau$

Supposing the equivalent input noise bandwidth is $B_{w}$, and its power spectrum density function is $N_{0}$, then the input noise power is:

$P_{\mathrm{ni}}=N_{0} B_{w}$

The input useful signal power is:

$P_{\mathrm{si}}=\frac{1}{2} Y_{\mathrm{D}}^{2} \varepsilon_{\mathrm{d}}^{2} \cdot \mathrm{V}^{2}$ (V is unit)

The output useful signal power is:

$P_{\text {so }}=\left(\frac{1}{C_{\mathrm{I}}} V_{\text {ref }} Y_{\mathrm{D}} \varepsilon_{\mathrm{d}} T_{\mathrm{I}}\right)^{2}$

So the system's SNIR is:

$S N I R=\frac{S N R_{o}}{S N R_{i}}=\frac{P_{s o}}{P_{n o}} / \frac{P_{s i}}{P_{n i}}=\frac{V_{\text {ref }}^{2} T_{\mathrm{I}} B_{w}}{2 V^{2}}$

$T_{\mathrm{I}}$ in equation (12) could be reduced as far as possible to achieve rapid detection, which also reduces the measurement time of the system when achieving the SNIR requested.

In order to compare rapid cross-correlation detection with traditional LIA detection, MDS is defined as the minimum detectable input signal of the system when system's output power SNR is

$S N R_{o}=\frac{P_{s o}}{P_{n o}}=\frac{V_{s o}{ }^{2}}{V_{n o}{ }^{2}}=\frac{(A \cdot M D S)^{2}}{\left(A \cdot \overline{V_{n i}}\right)^{2}}=1$

Where $\overline{V_{\mathrm{n}}}$ is the equivalent input noise of the system, $A$ is the gain of system.

Hence, $\overline{V_{\mathrm{ni}}}=M D S$.

The aim of weak signal detection methods is reducing the input equivalent noise level of the detective method (improving the ability of restraining noise of detective method).

\section{Experimental Results}

The prototype of the testing system for resonant pressure sensors based on rapid cross-correlation detection has been designed. The results of open-loop test using rapid cross-correlation detection and 
traditional LIA are shown in Fig.5 and Table1.

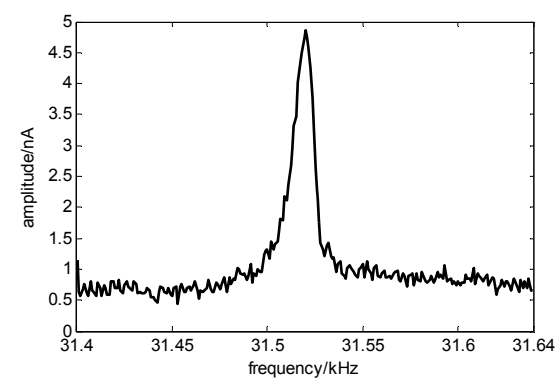

a) rapid cross-correlation detection

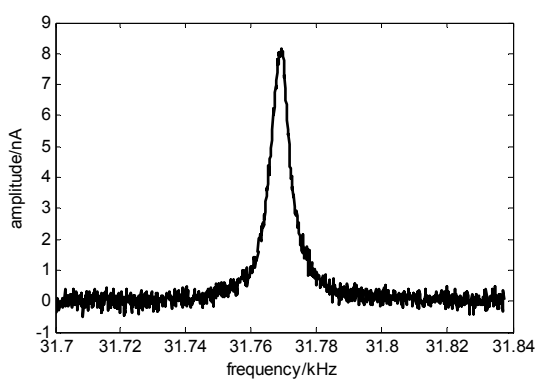

b) traditional LIA

Fig.5 Output amplitude- frequency characteristic

Table 1 Experimental results of two methods comparing

\begin{tabular}{ccc}
\hline methods & MDS $(\mathrm{nA})$ & $\begin{array}{c}\text { measuring time for } \\
\text { each frequency point }(\mathrm{ms})\end{array}$ \\
\hline $\begin{array}{c}\text { rapid cross-correlation } \\
\text { traditional LIA }\end{array}$ & 0.03 & 4 \\
& 0.1 & 100 \\
\hline
\end{tabular}

It can be seen from Table 1 that the rapid cross-correlation detection has larger SNIR when its measurement time is $1 / 25$ of the traditional LIA. So the efficiency of the measurement increases greatly when rapid cross-correlation is applied to the resonant pressure sensor.

\section{Summary}

A novel rapid cross-correlation detection method for micro-machined resonant pressure sensors has been presented, and the experimental results show that SNIR of rapid detection method is larger for the same measurement time. So the rapid cross-correlation detection could obtain required SNIR with the shorter time, which is so called "rapid".

\section{References}

[1]. Hutomo Suryo Wasisto, Stephan Merzsch, Andreas Waag, et al. Airborne engineered nanoparticle mass sensor based on a silicon resonant cantilever. Sensors \& Actuators: B. Chemical, Vol. 180 (2013) No. 10, p. 77-89.

[2]. Gao J Z. Detection of weak signals. Beijing: Tsinghua University press, 2004.

[3]. Hu A, Vamsy P. CMOS Optoelectronic Lock-in amplifier with integrated phototransistor array. IEEE Transactions on Biomedical Circuits and Systems. Vol. 4 (2010) No. 5, p. 274-280.

[4]. Davies R, Meuli G. Development of a digital lock-in amplifier for open-path light scattering measurement. 2010 IEEE Symposium on Industrial Electronics and Application.2010, p. 50-55.

[5]. Patel A, Kosko B. Noise Benefits in quantizer-array correlation detection and watermark decoding. IEEE Transactions on Signal Processing, Vol. 59 (2011) No. 2, p. 488-500.

[6]. Lin I S, Weiner A M. Selective correlation detection of photonically generated ultrawideband RF Signals. IEEE Journal of Lightwave Technology. Vol. 26 (2008) No. 15, p. 2692-2699.

[7]. Hida T, Kuo H H, Potthoff J, et al. White noise-an infinite dimensional calculus. Kluwer Academic Publ. 1993.

[8]. Fabing Duan, François Chapeau-Blondeau, Derek Abbott. Non-Gaussian noise benefits for coherent detection of narrowband weak signal. Physics Letters A, Vol. 378 (2014) No. 26-27, p. 1820-1824. 